\title{
Choroidal thickness changes and choriocapillary circulation analysis in macular holes using optical coherence tomography angiography
}

\author{
Nalei Zhou, Xiaolei Han, Enliang Ding, Yuhua Hao \\ Department of Ophthalmology, the Second Hospital of Hebei Medical University, Shijiazhuang, China \\ Contributions: (I) Conception and design: Y Hao, X Han; (II) Administrative support: Y Hao; (III) Provision of study materials or patients: X Han, E \\ Ding; (IV) Collection and assembly of data: N Zhou, E Ding; (V) Data analysis and interpretation: N Zhou; (VI) Manuscript writing: All authors; (VII) \\ Final approval of manuscript: All authors. \\ Correspondence to: Yuhua Hao. Department of Ophthalmology, the Second Hospital of Hebei Medical University, No. 215, West Heping Road, \\ Shijiazhuang 050000, China. Email: haoyuhua2019@126.com.
}

\begin{abstract}
Background: To investigate the changes of subfoveal choroidal thickness (SFCT) and choriocapillary circulation in idiopathic macular holes (IMHs), the fellow eyes, and normal subjects using optical coherence tomography (OCT) and optical coherence tomography angiography (OCTA), and to explore the role of SFCT and choriocapillary circulation in the pathogenesis of IMH.

Methods: A case series of 30 patients with unilateral IMH and 30 subjects, with age- and sex-matched, were included. Thirty eyes of 30 patients with unilateral IMH, the unaffected fellow eyes, and 30 eyes of age- and sex-matched healthy patients were studied. SFCT was measured by spectral domain enhanced depth imaging optical coherence tomography (EDI SD-OCT), while vascular density and blood flow area of choriocapillary circulation were obtained by OCTA.

Results: The SECT in IMH eyes $(236.09 \pm 79.25 \mu \mathrm{m})$ was lower than that in the unaffected fellow eyes $(249.71 \pm 86.10 \mu \mathrm{m})(\mathrm{P}=0.040)$ and healthy control eyes $(283.29 \pm 64.16 \mu \mathrm{m})(\mathrm{P}=0.001)$. Also, the SFCT of the unaffected fellow eyes was lower than that of the healthy control eyes $(\mathrm{P}=0.033)$. The superficial blood flow area and vascular density of choriocapillary in the macular area were smaller and lower in the IMH eyes $\left(2.84 \pm 0.35 \mathrm{~mm}^{2}, 20.74 \% \pm 8.26 \%\right)$ than the unaffected fellow eyes $\left(3.19 \pm 0.23 \mathrm{~mm}^{2}, 35.18 \% \pm 5.20 \%\right)$ and healthy control eyes $\left(3.26 \pm 0.24 \mathrm{~mm}^{2}, 35.20 \% \pm 6.49 \%\right)(\mathrm{P}<0.001)$; however, no difference was observed between the unaffected fellow eyes and healthy control eyes.

Conclusions: The SFCT in IMH eyes was lower than that of unaffected fellow eyes and matched control eyes. In addition, the choriocapillary blood flow area and vascular density were smaller and lower than those of the unaffected fellow eyes and normal controls. Our findings suggested that atrophy of choriocapillary might play an important role in the formation of IMH.
\end{abstract}

Keywords: Idiopathic macular hole (IMH); optical coherence tomography (OCT); optical coherence tomography angiography (OCTA); choriocapillaris; vascular density

Submitted Oct 05, 2021. Accepted for publication Dec 06, 2021.

doi: 10.21037/apm-21-3257

View this article at: https://dx.doi.org/10.21037/apm-21-3257

\section{Introduction}

Idiopathic macular hole (IMH) refers to the complete defect of the neuroretina, which can cause severe vision loss in elderly patients (1). The prevalence of full-thickness macular hole is 1.6 out of 1,000 elderly Chinese in northern China (2). The annual incidence of IMH is 9.2 eyes per 100,000 people in China, and 8.69 eyes per 100,000 people in the US $(2,3)$. The most recognized risk factors 
for IMH are age and sex. It has been proposed that IMH is the result of tangential traction exerted by contracted cortical vitreous. However, some IMH does not combine with posterior vitreous detachment, which indicates no tangential traction. The nutrition for macula is provided exclusively by choriocapillaris. Recently, some studies have shown that subfoveal choroidal thickness (SFCT) is significantly reduced. Hamzah $e t$ al. revealed that the deep foveal avascular zone area was more enlarged in IMH eyes before surgery (4). It remains controversial whether SFCT and choriocapillaris may influence the formation of IMH. Maybe the atrophy of choroid would cause the deficiency of nutrition for macula. With the tangential traction, finally these two factors cause the formation of IMH.

It is impossible to investigate the detail of choriocapillaris with conventional equipment. Optical coherence tomography angiography (OCTA) is the most reliable OCT for the qualification and quantification of retinal blood vessels. By comparing and calculating the changes of blood flow signals within blood vessels at different times, we can distinguish blood vessels from static background. Compared with traditional indocyanine green angiography, OCTA can detect very subtle changes of retinal and choroidal blood flow, without any influence of indocyanine green leakages. Previous articles reported either about the choroidal thickness changes or choroidal blood circulation. We investigated both the choroidal thickness changes and choroidal blood circulation at same time with more cases.

In this study, we investigated the SFCT, flow area, as well as the vascular density of choriocapillaris of the macula in $\mathrm{IMH}$, and compared these with unaffected fellow eyes and matched control eyes to identify the formation of IMH as well as potential avenues for preventing and treating IMH. We present the following article in accordance with the STROBE reporting checklist (available at https://dx.doi. org/10.21037/apm-21-3257).

\section{Methods}

\section{Patients}

The study was conducted in accordance with the Declaration of Helsinki (as revised in 2013) and was approved by the institutional review board of the Second Hospital of Hebei Medical University (approval number: 2018-P052). With clear and full explanations of the aim of our research, all subjects signed the informed consent forms. In total, 30 patients with unilateral IMH (22 females and eight males, stages 2-4), and 30 age- and sex-matched healthy controls were enrolled. The diagnosis of IMH was confirmed using OCT (Heiderberg, Germany), and FFA was used to rule out secondary macular hole. The stage of IMH was classified according to the definition given by Gass. The healthy controls were age- and sex-matched, and their best corrected visual acuity (BCVA) was better than 0.9 without ocular disease.

All subjects underwent thorough ocular examination including BCVA, non-contact tonometry, fundus photography, axial length (AL), OCT, and OCTA. The exclusion criteria for all subjects were as follows: (I) AL $>26 \mathrm{~mm}$; (II) refractive error >-6.00 D; (III) intraocular pressure (IOP) $>22 \mathrm{mmHg}$; (IV) patients with a history of retinal disease; (V) patients who had undergone retinal surgery or other treatment; and (VI) patients with ocular trauma or tumor, glaucoma, uveitis, severe media opacity, or unstable fixation.

\section{OCT acquisition}

The SFCT was obtained using the spectral-domain OCT system (Heidelberg, Germany). According to the instructions provided by the manufacturer, we used the raster mode to scan the macula with scan line length of $8.8 \mathrm{~mm}$, centered on the fovea. The vertical line between the outer margin of RPE and the inner margin of sclera was draw, and the distance between these two layers was recorded. The SFCT was the vertical distance between the outer margin of the retinal pigment epithelium (RPE) layer and the inner face of sclera (Figure 1). All measurements were independently measured by two experienced doctors. The data were measured three times, and the average value was taken as the final SFCT.

\section{OCTA acquisition}

OCT RS-3000 angiography (NIDEK, Japan) was used to record the blood flow area and vascular density of the choriocapillaris layer of the macula. The angio-retina scan mode was chosen, with a scan area of $3 \mathrm{~mm} \times 3 \mathrm{~mm}$, centered just at the fovea. The measurement of blood flow area and vascular density within $1 \mathrm{~mm}$ (radius) was automatically measured using the OCT RS-3000 software (Figure 2).

\section{Statistical analysis}

All analyses were performed using SPSS 24.0 (IBM-SPSS, 

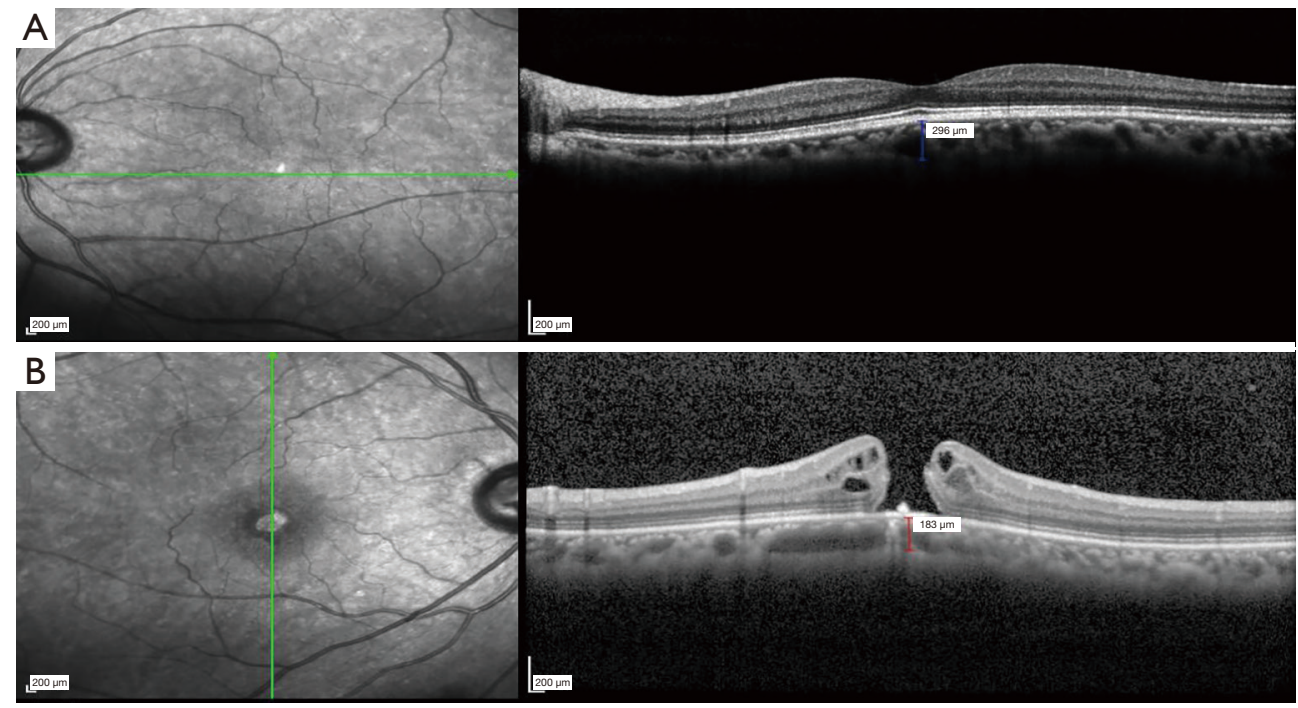

Figure 1 Cross-sectional imaging of the choroid using enhanced depth imaging spectral-domain optical coherence tomography (EDI SDOCT). The vertical distance between the outer boundary of the retinal pigment layer and the inner boundary of the sclera boundary was used to represent the thickness of choroid. (A) The unaffected fellow eye; (B) IMH. IMH, idiopathic macular hole.
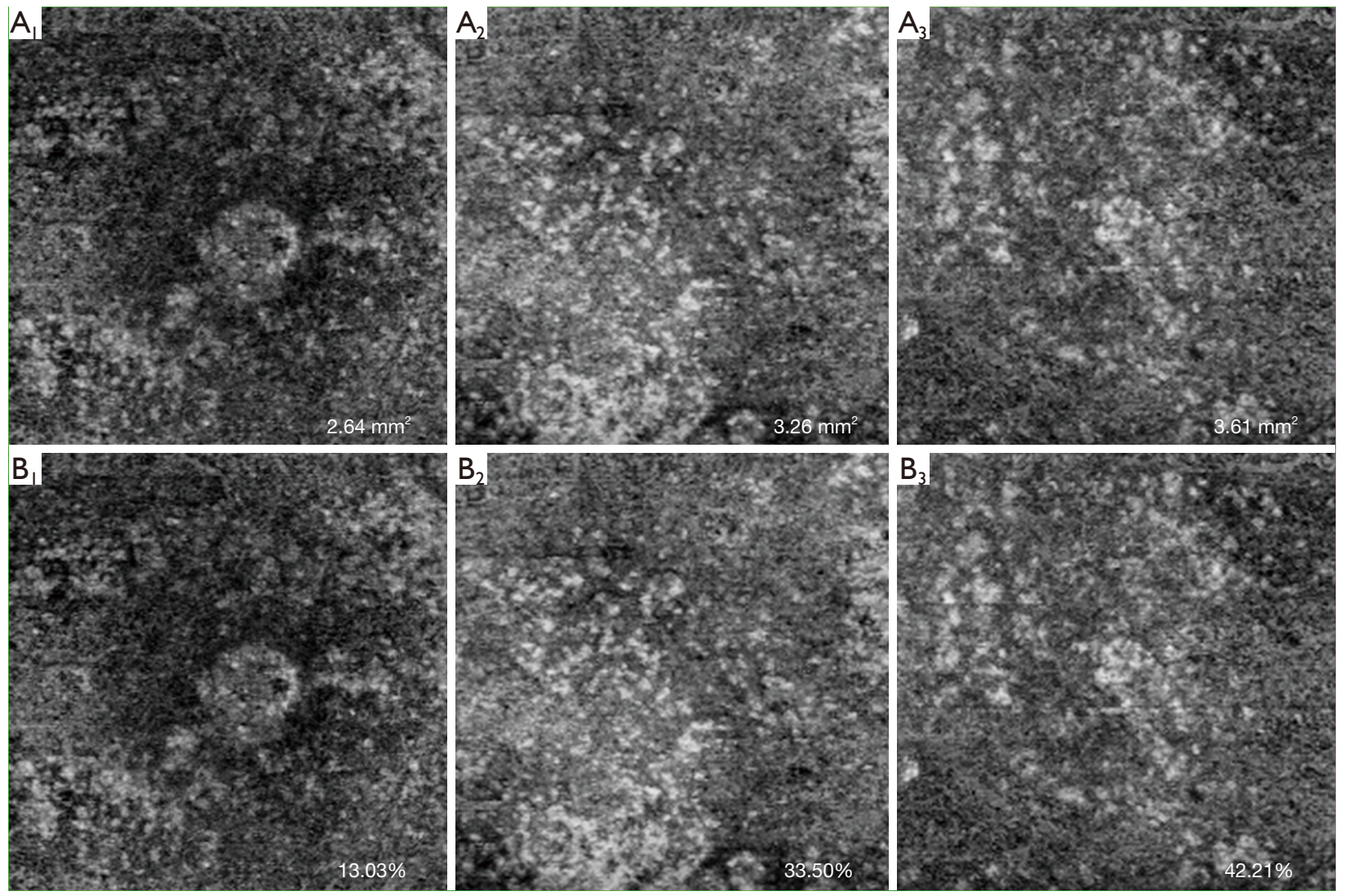

Figure 2 OCTA image. $\mathrm{A}_{1}-\mathrm{A}_{3}$ show the flow area in IMH eyes, unaffected eyes, and healthy eyes of the choriocapillaris, respectively. $\mathrm{B}_{1}-$ $\mathrm{B}_{3}$ display the vascular density in IMH eyes, unaffected eyes, and healthy eyes of the choriocapillaris, respectively. OCTA, optical coherence tomography angiography; IMH, idiopathic macular hole. 
Table 1 General data of the IMH eyes, unaffected fellow eyes, and matched controls

\begin{tabular}{|c|c|c|c|c|c|c|}
\hline Basic information & \multicolumn{3}{|c|}{ Patients } & \multicolumn{3}{|c|}{$P$ value } \\
\hline Gender (male/female) & $22 / 8$ & $22 / 8$ & $22 / 8$ & & 1.000 & 1.000 \\
\hline Age (years $\pm S D$ ) & $62 \pm 11$ & $62 \pm 11$ & $64 \pm 11$ & & 0.492 & 0.492 \\
\hline Axial length (mm) & $23.21 \pm 0.74$ & $23.41 \pm 0.48$ & $23.36 \pm 0.59$ & 0.204 & 0.0388 & 0.692 \\
\hline Duration (months) & $5.53 \pm 3.09$ & & & & & \\
\hline
\end{tabular}

$\mathrm{IMH}$, idiopathic macular hole; IOP, intraocular pressure.

Chicago, Illinois, USA). Continuous data were recorded as the mean \pm standard deviation, and categorical variables were recorded as percentages. Comparisons between the $\mathrm{IMH}$ and unaffected fellow eyes were analyzed using the paired $t$-test. Comparisons between the IMH and healthy controls, unaffected fellow eyes, and healthy controls were analyzed by repeated measurement analysis of variance. A $P$ value less than 0.05 was considered statistically significant.

\section{Results}

\section{Basic information of the patients}

According to the inclusion criteria and exclusion criteria, 30 cases of unilateral IMH diagnosed by OCT were included in this study, including eight males and 22 females. The mean age of the patients was $(62 \pm 11)$ years, and the onset time was $(5.53 \pm 3.09)$ months. Thirty ageand sex-matched healthy controls were also recruited. There was no significant difference in the axial length (AL) $(\mathrm{F}=0.907)$ and IOP $(\mathrm{F}=0.591)$ among these three groups $(\mathrm{P}>0.05)$ (Table 1).

\section{Comparison of SFCT among the different groups}

The SFCT in the IMH, unaffected fellow eyes, and matched controls were $236.09 \pm 79.25,249.71 \pm 86.10$, and $283.29 \pm 64.16 \mu \mathrm{m}$, respectively. The SFCT of IMH eyes was lower than that of the unaffected fellow eyes and the healthy controls ( $\mathrm{F}=8.837, \mathrm{P}=0.040,0.000)$, while the SFCT of the unaffected fellow eyes was lower than that of the healthy controls $(\mathrm{F}=8.837, \mathrm{P}=0.033)$.

\section{Comparison of superficial blood flow area and vascular density of choriocapillaris}

The superficial blood flow area of the choriocapillaris in the IMH, unaffected fellow eyes, and matched controls were $2.84 \pm 0.35,3.19 \pm 0.23$, and $3.26 \pm 0.24 \mathrm{~mm}^{2}$, respectively. The superficial blood flow area of the choriocapillaris in IMH eyes was lower than that of the unaffected fellow eyes and the matched controls $(\mathrm{F}=19.768, \mathrm{P}=0.000,0.000)$. There was no significant difference between the flow area in unaffected fellow eyes and that of healthy controls $(\mathrm{F}=19.768, \mathrm{P}=0.332)$. The superficial vascular density of the choriocapillaris in the IMH, unaffected fellow eyes, and matched controls were $(20.74 \pm 8.26) \%,(35.18 \pm 5.20) \%$, and $(35.20 \pm 6.49) \%$, respectively. The superficial vascular density of the choriocapillary in IMH eyes was lower than that in the unaffected fellow eyes and the healthy controls $(\mathrm{F}=45.583, \mathrm{P}=0.000,0.000<0.05)$. There was no significant difference between the vascular density in the unaffected fellow eyes and that of the healthy controls $(F=45.583$, $\mathrm{P}=0.994>0.05$ ) (Table 2, Figure 3).

\section{Discussion}

The fovea is nourished by the choroidal circulation, and therefore, choroidal blood perfusion plays important role in macular pathophysiology. Various factors, such as advanced age and ocular diseases, can lead to a decrease in the volume of blood flow to the choroid, which may lead to a decrease in the SFCT, as well as a decrease in blood flow area and vascular density (5-7).

In this study, our results showed that the SFCT of the healthy controls, unaffected fellow eyes, and IMH eyes 
Table 2 Comparison of the SFCT and choroidal circulation in IMH eyes, unaffected fellow eyes, and healthy controls

\begin{tabular}{|c|c|c|c|c|c|c|c|}
\hline Choroidal parameters & \multicolumn{4}{|c|}{ Patients } & \multicolumn{3}{|c|}{$P$ value } \\
\hline SFCT $(\mu \mathrm{m})$ & $224.66 \pm 71.71$ & $253.29 \pm 84.14$ & $283.29 \pm 64.17$ & 8.837 & 0.040 & 0.000 & 0.033 \\
\hline Blood flow area $\left(\mathrm{mm}^{2}\right)$ & $2.84 \pm 0.35$ & $3.19 \pm 0.23$ & $3.26 \pm 0.24$ & 19.768 & 0.000 & 0.000 & 0.332 \\
\hline
\end{tabular}

SFCT, subfoveal choroidal thickness; IMH, idiopathic macular hole.
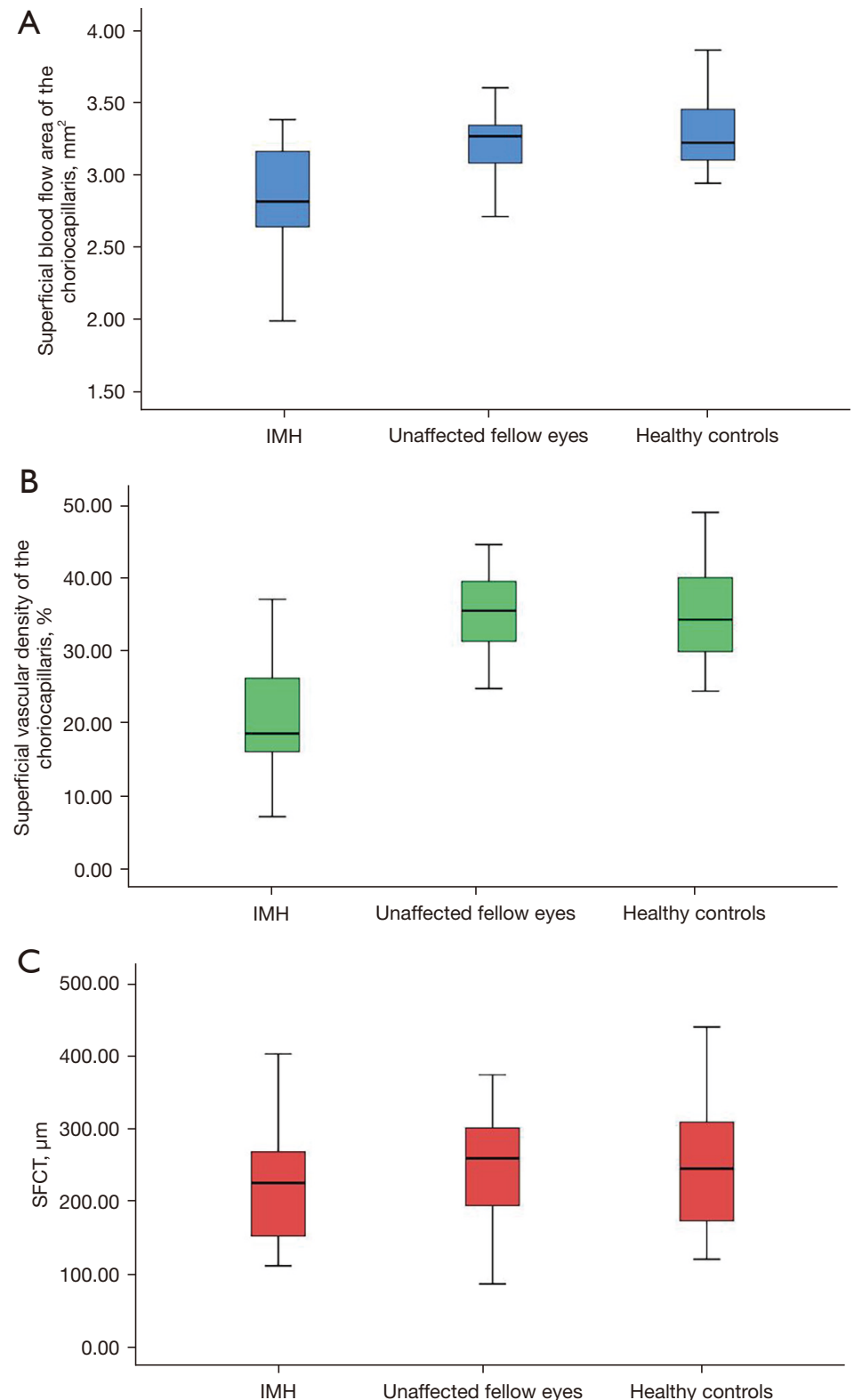

Figure 3 Comparisons of the choriocapillary blood flow area, vascular density, and SFCT in IMH, unaffected fellow eyes, and matched controls. SFCT, sub-foveal choroidal thickness; IMH, idiopathic macular hole. 
decreased one-by-one fractionally. It is suggested that the pathogenesis of IMH may be related to choroidal thickness and the circulation of macular choriocapillaris. It is known that the nutrition for the macula area is mainly provided by choriocapillaris. With age, the SFCT and blood flow of the choriocapillaris decreases, which can cause dystrophy as well as the loss of retinal tissue in the macular area. Ultimately, macular holes are the result of tangential traction of vitreous on the vulnerable retinal tissue. Right now, IMH couldn't be prevented, the only thing we can do is early diagnosis of IMH and early treatment for IMH. For IMH without vitreomacular adhesion, vitrectomy is the only option. It's reported that in smaller IMH with vitreomacular adhesion, ocriplasmin has a greater success rate.

The superficial blood flow area and blood flow density of the choriocapillaris at the macula of IMH eyes were lower than those in the unaffected fellow eyes and healthy controls; however, there was no significant difference between those of the unaffected fellow eyes and healthy controls. Also, the choroidal thickness of the unaffected fellow eyes of IMH patients was lower than that of the healthy controls, but the capillary blood flow area and blood flow density in the choriocapillaris of the unaffected fellow eyes were not significantly different from those of the healthy controls, which may be due to the self-regulation of the choroidal vessels $(8,9)$.

The full choroidal thickness can be obtained by SDOCT, including large and medium choroidal vessels, and choriocapillaris, while OCT-A can only detect superficial choriocapillaris. The SFCT decreases with age, which can lead to a decrease in the volume of blood flow to the choroid. When the perfusion pressure of large and medium choroidal vessels in IMH patients decreases, automatic regulation mechanism of the choroidal vessels may be initiated, thereby maintaining the capillary blood flow of the choroid at relatively normal levels, which could also explain why there was no significant difference between the unaffected fellow eyes and the healthy controls.

Our study included multimodal imaging of IMH with OCT and OCTA, and analyzed the changes of SFCT and blood flow parameters of choriocapillaris at the same time. This is the advantage of our study. Our study had some limitations that should be noted. Firstly, our limitation is the relatively small number of patient recruited. Secondly, further investigation should be conducted to verify the relationship between SFCT and blood flow parameters of choriocapillaris, which is under investigation by our team.

In conclusion, changes in choriocapillary circulation may play a role in the occurrence of IMH. However, a large number of pre- and post-operative comparative studies are needed.

\section{Acknowledgments}

Funding: This project was supported by Hebei Medical applicable technology tracking project (G2019054) and Natural Science Foundation of Hebei Province (H2020206645).

\section{Footnote}

Reporting Checklist: The authors have completed the STROBE reporting checklist. Available at https://dx.doi. org/10.21037/apm-21-3257

Data Sharing Statement: Available at https://dx.doi. org/10.21037/apm-21-3257

Conflicts of Interest: All authors have completed the ICMJE uniform disclosure form (available at https://dx.doi. org/10.21037/apm-21-3257). YH reports this project was supported by Hebei Medical applicable technology tracking project (G2019054) and Natural Science Foundation of Hebei Province (H2020206645). The other authors have no conflicts of interest to declare.

Ethical Statement: The authors are accountable for all aspects of the work in ensuring that questions related to the accuracy or integrity of any part of the work are appropriately investigated and resolved. The study was conducted in accordance with the Declaration of Helsinki (as revised in 2013) and was approved by the institutional review board of the Second Hospital of Hebei Medical University (approval number: 2018-P052). With clear and full explanations of the aim of our research, all subjects signed the informed consent forms.

Open Access Statement: This is an Open Access article distributed in accordance with the Creative Commons Attribution-NonCommercial-NoDerivs 4.0 International License (CC BY-NC-ND 4.0), which permits the noncommercial replication and distribution of the article with the strict proviso that no changes or edits are made and the original work is properly cited (including links to both the formal publication through the relevant DOI and the license). See: https://creativecommons.org/licenses/by-nc-nd/4.0/. 


\section{References}

1. Ali FS, Stein JD, Blachley TS, et al. Incidence of and Risk Factors for Developing Idiopathic Macular Hole Among a Diverse Group of Patients Throughout the United States. JAMA Ophthalmol 2017;135:299-305.

2. Wang S, Xu L, Jonas JB. Prevalence of full-thickness macular holes in urban and rural adult Chinese: the Beijing Eye Study. Am J Ophthalmol 2006;141:589-91.

3. McCannel CA, Ensminger JL, Diehl NN, et al. Population-based incidence of macular holes. Ophthalmology 2009;116:1366-9.

4. Hamzah F, Shinojima A, Nakashizuka H, et al. Foveal Avascular Zone Area Analysis in Macular Hole Before and After Surgery Using Optical Coherence Tomography Angiography. Ophthalmic Surg Lasers Imaging Retina 2018;49:329-35.

5. Tsuiki E, Suzuma K, Ueki R, et al. Enhanced depth

Cite this article as: Zhou N, Han X, Ding E, Hao Y. Choroidal thickness changes and choriocapillary circulation analysis in macular holes using optical coherence tomography angiography. Ann Palliat Med 2021;10(12):12367-12373. doi: 10.21037/apm-21-3257 imaging optical coherence tomography of the choroid in central retinal vein occlusion. Am J Ophthalmol 2013;156:543-7.e1.

6. Ruiz-Moreno JM, Staicu C, Pinero DP, et al. Optical coherence tomography predictive factors for macular hole surgery outcome. Br J Ophthalmol 2008;92:640-4.

7. Maruko I, Iida T, Sugano Y, et al. Subfoveal choroidal thickness after treatment of Vogt-Koyanagi-Harada disease. Retina 2011;31:510-7.

8. Chen $\mathrm{H}, \mathrm{Wu} \mathrm{Z}$, Chen $\mathrm{Y}$, et al. Short-term changes of choroidal vascular structures after phacoemulsification surgery. BMC Ophthalmol 2018;18:81.

9. Nagaoka T. Physiological mechanism for the regulation of ocular circulation. Nippon Ganka Gakkai Zasshi 2006;110:872-8.

(English Language Editor: A. Kassem) 